\title{
Strong correlation effects in the $(5,5) @(10,10)$ double-walled carbon nanotubes in the framework of the $t-J$ model
}

\author{
E. Rostampour ${ }^{1}$
}

Published online: 2 June 2016

(C) The Author(s) 2016. This article is published with open access at Springerlink.com

\begin{abstract}
Double-walled carbon nanotubes are coaxial nanostructures composed of exactly two single-walled carbon nanotubes, one nested in another. This unique structure offers advantages and opportunities for extending our knowledge and application of the carbon nanomaterials family. An emphasis is placed on the double wall physics that contributes to these structures' complex inter-wall coupling of electronic and optical properties. The susceptibility tensor of doublewalled carbon nanotubes of the "armchair" type the ABAB packing of layers is investigated theoretically in the weak and strong correlation regime. We calculated the spin and charge susceptibility tensor for of the $(5,5) @(10,10)$ double-walled carbon nanotubes using the random-phase approximation in the weak correlation regime. The relationship with the Heisenberg limit (large U/t) is discussed. Our results show that the susceptibility spectra of the $(5,5) @(10,10)$ doublewalled carbon nanotubes shift peaks to lower energies with increase in the correlation parameter of the $\mathrm{t}-\mathrm{J}$ model $(U \geq$ $t)$. These results show that electronic correlation effects should be taken into account in theoretical studies of doublewalled carbon nanotubes. We discuss the relevance of our results for the $\mathrm{C}_{20}$ and $\mathrm{C}_{60}$ fullerens and $(5,5)$ nanotubes.
\end{abstract}

Keywords Strong correlation effects · The $(5,5) @(10$, 10) double-walled carbon nanotubes $\cdot$ T-J model

E. Rostampour

el_rostampour@yahoo.com

1 Young Researchers and Elites club, Science and Research Branch, Islamic Azad University, Tehran, Iran

\section{Introduction}

Double-walled carbon nanotubes (DWCNTs) are intriguing materials that exhibit properties intermediate between those of single-walled carbon nanotubes (SWCNTs) and multiwalled carbon nanotubes (MWCNTs). These carbon nanomaterials formed from two concentric cylinders of graphene can be routinely synthesized with outer-wall diameters below $2 \mathrm{~nm}$, and they exhibit band gaps that are sufficiently large for use in field-effect transistors [1-3]. The state of the art in modeling and simulation of nanostrucutred materials and systems are studied. Vibrations of DWCNTs have considered in several papers. The researcher studied the free vibrations of a DWCNT which composed of two coaxial SWCNTs interacting each other by the interlayer van der Waals forces. Therefore, the inner and outer carbon nanotubes are modeled as two individual elastic beams. In addition, the outer wall can be functionalized without changing the mechanical and electronic properties of the inner tube [4]. Compared to SWCNTs, DWCNTs have higher mechanical strength and thermal stability and they also possess interesting electronic and optical properties. Although DWCNTs were discovered in 1991 and the first synthesis was reported in 1998, this structure has received relatively little attention until the recent synthesis [5] and separation [6] of high purity samples. DWCNT (the simplest MWCNT) has utilized as an ideal theoretical model for studying the interaction and coupling behaviors between concentric tubes [7-10]. Unfortunately, there is no experimental evidence for understanding intertube interactions, because it is not possible to produce pure samples of DWCNTs (with $100 \%$ purity and the total absence of SWCNTs) using presently available synthesis techniques. Even though some photoluminescence studies have reported for catalytically grown DWCNTs containing small fractions of SWCNTs [11] and high purity 
of DWCNTs (ca. $90 \%$ ) using the selective removal of SWCNTs via oxidation treatments [12], it is not easy to completely discard the effects of SWCNTs as impurity. In the fluorination process of DWCNTs, mainly the outer tube is fluorinated, and the inner tube remains almost unaffected by fluorine atoms. When compared to SWCNTs synthesized with the chemical vapor deposition process, the inner tube of a DWCNT has a smaller diameter distribution due to the confinement and interaction with the outer tube that limits the diameter of the inner tube. The early theoretical studies of the electronic properties of single-walled carbon nanotubes predicted that SWCNTs could be either metallic or semiconducting depending on their structural parameters [13-15]. In the $\pi$-tight-binding model within the zone-folding scheme, one third of the nanotubes are metallic and two thirds are semiconducting depending on their indices (n, $\mathrm{m}$ ). The tightbinding calculations based on the use of $\sigma$ and $\pi$ bands, due to the curvature-induced mixing of these bands, predict that some metallic nanotubes to be very-small-gap semiconducting nanotubes $[14,15]$. In MWCNTs and in bundles of SWCNTs the lower symmetry of these structures with respect to the symmetry of the separate SWCNTs leads to the appearance or disappearance of the band gap [16,17]. Our approach, which is outlined in Sect. 2, is based on a numerical method of Heeger et al. which was originally developed to treat correlation effects in many-body calculations of atoms. In Sect. 3, calculation results presented and discussed in detail the susceptibility tensor and Correlation effects of the $(5,5) @(10,10)$ double-walled carbon nanotubes. Finally, in Sect. 4, we summarize our results.

\section{Model and method}

Hubbard model and its variants constitute an important research topic in theoretical condensed matter physics, particularly in the context of strongly correlated electron systems. Most of the many-body techniques commonly used in condensed matter physics can be learnt in this context. Also there are some theoretical tools and concepts which apply to this model only. In order to describe the $(n, n)$ nanotubes, we utilize the extended Peierls-Hubbard Hamiltonian [18]

$$
\begin{aligned}
H_{n}= & -t_{i j n} \sum_{\langle i, j\rangle, \sigma}\left(c_{i \sigma}^{\dagger} c_{j \sigma}+H . c .\right)+\frac{U}{2} \\
& \times \sum_{i \sigma} c_{i \sigma}^{\dagger} c_{i \sigma} c_{i-\sigma}^{\dagger} c_{i-\sigma}+\frac{1}{2} K \sum_{\langle i, j\rangle}\left(y_{i j}\right)^{2} .
\end{aligned}
$$

where $c_{i \sigma}^{\dagger}\left(c_{i, \sigma}\right)$ is the creation (annihilation) operator of the $\pi$ electron at the site $i$ with spin $\sigma$, the sum is taken over nearest-neighbor pairs $\langle i, j\rangle, \mathrm{U}$ is the on-site electron correlation. Also, hopping integral at the bond $\langle i, j\rangle, t_{i j}$ is given by $t_{i j}=t_{0}+\alpha y_{i j}$ where $t_{0}, \alpha$ and $y_{i j}$ are hopping integral between nearest neighbor $i t h$ and $j t h$ sites of the ideal system, the electron-phonon coupling constant and the change of bond length between $i t h$ and $j t h$ sites of the ideal system, respectively. The term $c_{i \sigma}^{\dagger} c_{i \sigma} c_{i-\sigma}^{\dagger} c_{i-\sigma}$ is non-zero only when state $j$ has two electrons on it, one with spin $\uparrow$ and one with spin $\downarrow$.It is worth recalling that a simple tight-binding (TB) Hamiltonian for $\pi$ orbitals reproduces extremely well the order and degeneracies of electronic levels near the Fermi energy as obtained in more sophisticated molecular orbital calculation. For the whole range of $0<U / t<\infty$, we find that the pair-binding energy is always positive, ruling out the possibility of superconductivity induced solely by an electronic mechanism. With increasing $U / t$ there is, however, a very interesting metal-insulator transition predicted [19] to occur around $U / t \sim 4.1$ in molecular solids formed with the $\mathrm{C}_{20}$ fullerene. But antiferromagnetic correlations indicative of $\mathrm{N}$ order are present in these finite systems as clearly shown by numerical studies [20]. The ground state degeneracy that we expect due to broken symmetry appears only in the thermodynamic limit. For any finite system vector order parameters will be zero (such as the staggered magnetization of the square lattice magnet) but correlations can be properly studied through the use of singlet correlation function such as $\left\langle\mathrm{S}_{\mathrm{i}} \cdot \mathrm{S}_{\mathrm{j}}\right\rangle$. In the strong coupling regime $(U / t \gg 1)$ the half-filled Hubbard model reduces at leading order in perturbation theory to the antiferromagnetic Heisenberg model (HAF):

$H_{n H A F}=J \sum_{\langle i, j\rangle}\left(\mathrm{S}_{\mathrm{i}} \cdot \mathrm{S}_{\mathrm{j}}-\frac{1}{4}\right)$

where $\mathrm{S}_{\mathrm{i}}$ are spin-1/2 operators and $J=\frac{4 \mathrm{t}^{2}}{\mathrm{U}}$. To study this quantum model on finite clusters, one can search for the ground state of the corresponding classical model and perform the spin wave expansion around this state. This non-trivial ground state classical spin configuration has been recently discussed. For the quantum problem, a spin wave analysis has been performed, leading to a critical spin value (below which spin order is washed out by quantum fluctuations) rather close to $1 / 2$. This result was in rough agreement with more sophisticated QMC simulations [21,22]. The Hubbard Hamiltonian for the Multinanotube becomes that of the isolated layers with an inter-layer interaction term

$$
\begin{aligned}
& H_{\text {Multiwall carbon nanotubes }} \\
& \quad=\sum_{n}^{N} H_{n}+t_{\perp} \sum_{\langle i j\rangle}\left(c_{i}^{\dagger} c_{j}+c_{j}^{\dagger} c_{i}\right)
\end{aligned}
$$

where site $i$ belongs to a inner tube, and site $j$ is its NN or NNN on the adjacent outer tube and $t_{\perp}$ is the hopping between pairs of the NN and NNN between layers [23]. When interaction turns on, the spin $\left(\chi_{(S)}^{R P A}\right)$ or charge $\left(\chi_{(C)}^{R P A}\right)$ 


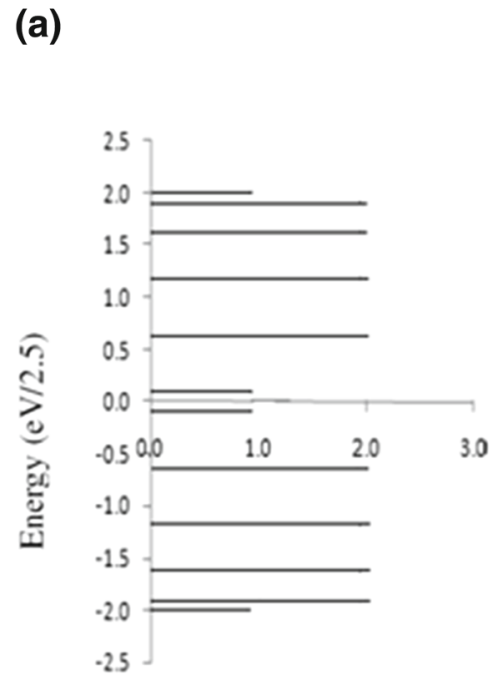

Degeneracy

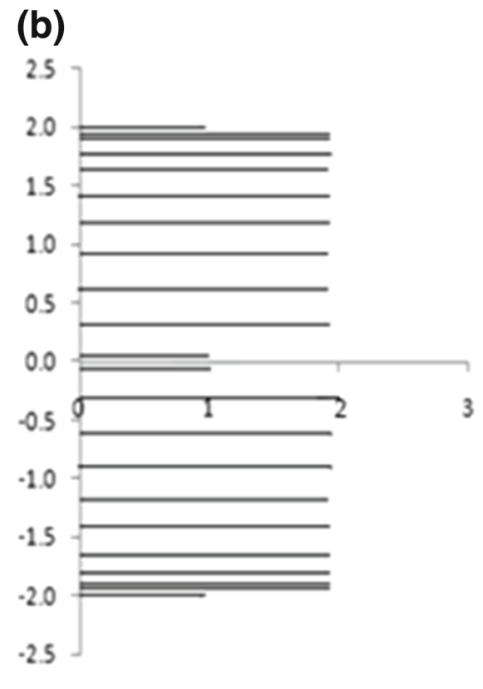

Degeneracy

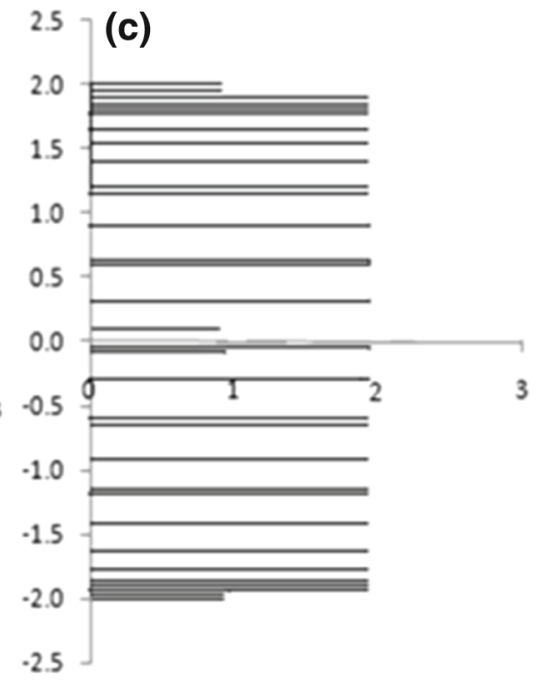

Degeneracy

Fig. 1 Energy levels of the $\mathbf{a}(5,5), \mathbf{b}(10,10)$ single-walled carbon nanotube and $\mathbf{c}$ of the $(5,5) @(10,10)$ double-walled carbon nanotubes in the TB regime $(\mathrm{U}=0)$

susceptibility of the $(5,5) @(10,10)$ double-walled carbon nanotubes in the random phase approximation (RPA) level is given by

$$
\begin{aligned}
& \chi_{(S)}^{R P A}=\frac{\chi^{0}}{\left[1-\mathrm{U} \chi^{0}\right]} \\
& \chi_{(C)}^{R P A}=\frac{\chi^{0}}{\left[1+\mathrm{U} \chi^{0}\right]}
\end{aligned}
$$

Where $\chi^{0}$ denotes the free susceptibility $(\mathrm{U}=0)$ of the model, and the instability occur when $\operatorname{Det}\left(1 \pm U \chi^{\wedge} 0(\omega=0)\right)=$ 0 . When $\mathrm{U}=0$ we have $\chi_{(C)}^{R P A}=\chi_{(s)}^{R P A}=\chi^{0}$. When the interaction strength $U$ is enhanced to a critical value $U_{c}$, the spin susceptibility of the model diverges, which implies the instability toward long-range spin density wave order [24].

\section{Results and discussions}

In the present theoretical study, we will perform a numerical calculation on the $(5,5) @(10,10)$ double-walled carbon nanotubes by usingthe Hubbard model. We set $t=2.5 \mathrm{eV}, \alpha=$ $6.31 \mathrm{eV} / \mathrm{A}^{\circ}, K=49.7 \mathrm{eV} / \mathrm{A}^{\circ 2}$ and $t_{\perp}=0 \mathrm{eV}$ in the calculations [25]. We calculate the energy levels of the $(5,5),(10,10)$ single-walled carbon nanotube and the $(5$, $5) @(10,10)$ double-walled carbon nanotubes with TB model to four decimal places with TB model that Fig. 1 shows the degeneracy of the energy levels. In graphite, the G mode is a single peak at around $1582 \mathrm{~cm}^{-1}$ with a full width at halfmaximum of $\sim 20 \mathrm{~cm}^{-1}$ at room temperature [26]. MWCNTs are often composed of more than ten nanotubes with diameters in the range of $10 \mathrm{~nm}$. Therefore, the MWCNT spectrum resembles the graphite spectrum rather than that of singlewalled carbon nanotubes. Because of the large diameters of the constituent tubes, radial breathing mode-like modes of MWCNT are usually too small in intensity and frequency to be detected. In Reference [27], it was shown that Raman modes in the RBM region can be observed in high-purity MWCNT samples if the diameter of the inner tube is smaller than $2 \mathrm{~nm}$. Also, the high-energy mode in MWCNT is very similar to the graphite $\mathrm{G}$ mode, mainly because of the large nanotube diameters. In contrast, DWCNTs typically consist of smaller-diameter tubes and have a Raman spectrum similar to that of SWCNTs [28-30]. The long-range part of interaction is essential in describing the excited state of $\mathrm{C}_{60}$. $U / t$ values for $\mathrm{C}_{20}$ isomers have been estimated by Lin and Sorensen, who show that the $\mathrm{C}_{20}$ fullerene cage is the most strongly correlated of the three major $\mathrm{C}_{20}$ isomers, with the on-site interaction strength as high as $U / t \sim 7.1$. The $\mathrm{C}_{20}$ fullerene cage is also the most strongly correlated molecule in the fullerene family, and is much more correlated than the $\mathrm{C}_{60}$ molecule, which has a $U / t$ ratio of approximately $U / t \sim 3.5$ [31]. Since $U / t \sim 7.1$, such a reduction is reasonable for the $\mathrm{C}_{20}$ cage. HAF defined on a dodecahedron molecule has been solved by exact diagonalization and shows a spin singlet and nonmagnetic ground state which agrees very well with our previous discussions [32]. Figure 2 shows the spin and charge susceptibility $\left(\chi=\frac{\chi_{x x}+\chi_{y y}+\chi_{z z}}{3}\right)$ of the $(5,5) @(10,10)$ double-walled carbon nanotubes in the random phase approximation. In the weak correlation $\left(\frac{U}{t}<1\right)$, the spin susceptibility is larger than the charge susceptibility 

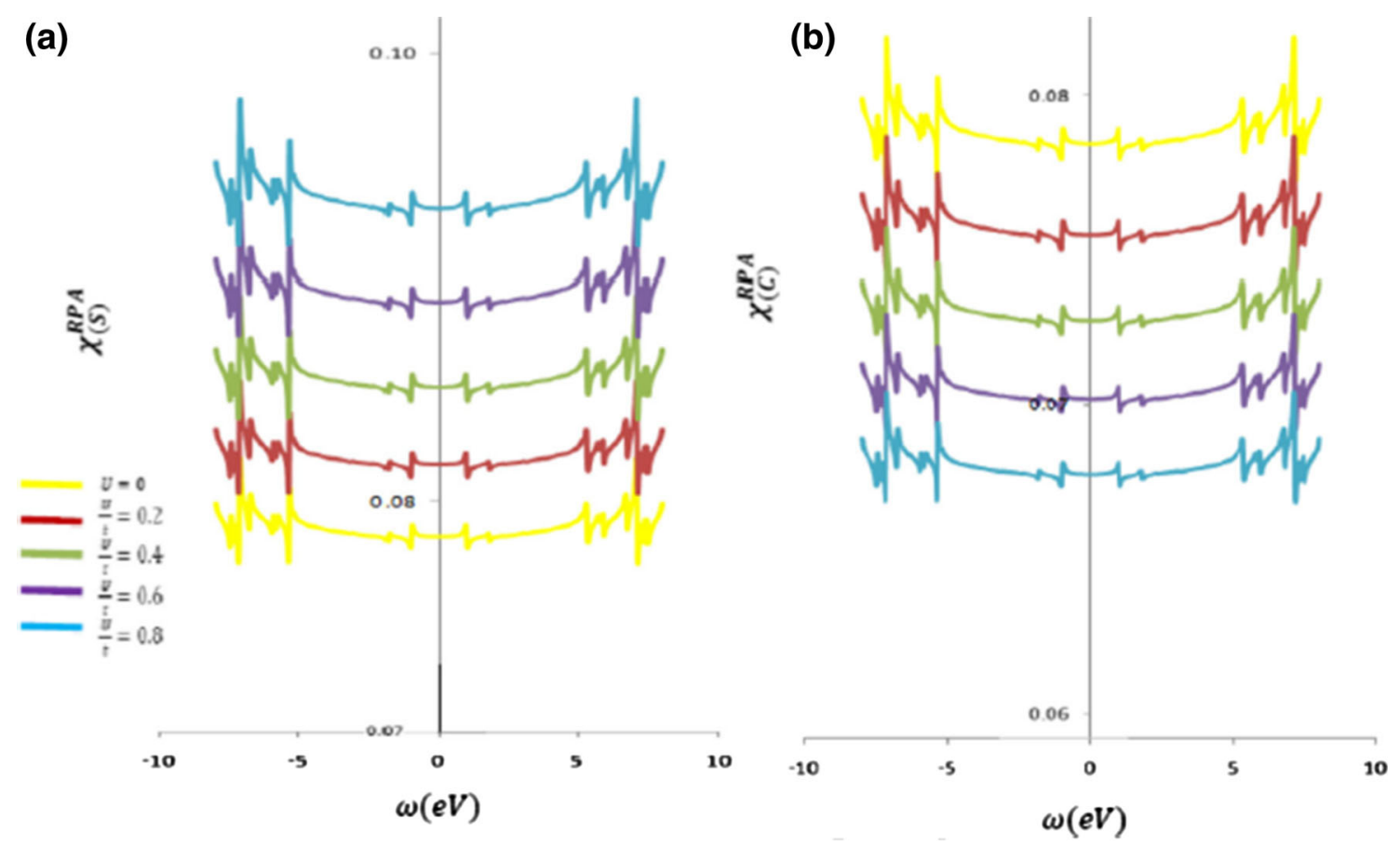

Fig. 2 The spin and charge susceptibility of the $(5,5) @(10,10)$ double-walled carbon nanotubes in the random phase approximation $($ Color figure online)
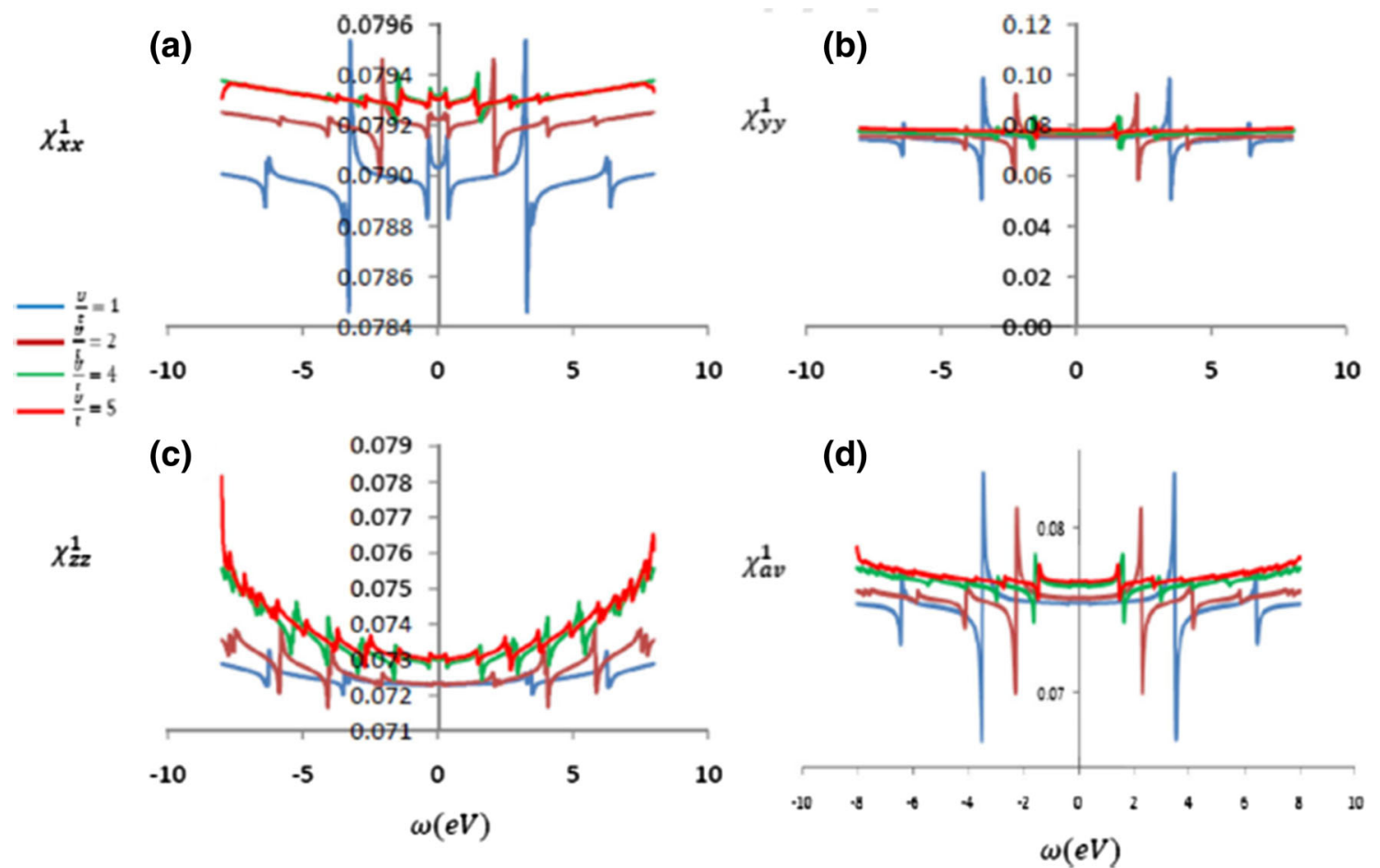

Fig. 3 The real part of the a $x x$, b $y y, \mathbf{c} z z$ elements of the susceptibility tensor, and $\mathbf{d}$ average of the susceptibility tensor for the (5, 5)@ (10,10) double-walled carbon nanotubes with $\mathrm{t}-\mathrm{J}$ model in the strong correlation (Color figure online)

for $(5,5) @(10,10)$. The spin susceptibility increases and the charge susceptibility decreases with increasing U Hubbard. The Hubbard model at large $U$ limit can be mapped into the so called $\mathrm{t}-\mathrm{J}$ model (the first term of eq.1 and eq. 2), where there are antiferromagnetic spin fluctuations along with hoppings restricted to subspace with no double occu- 


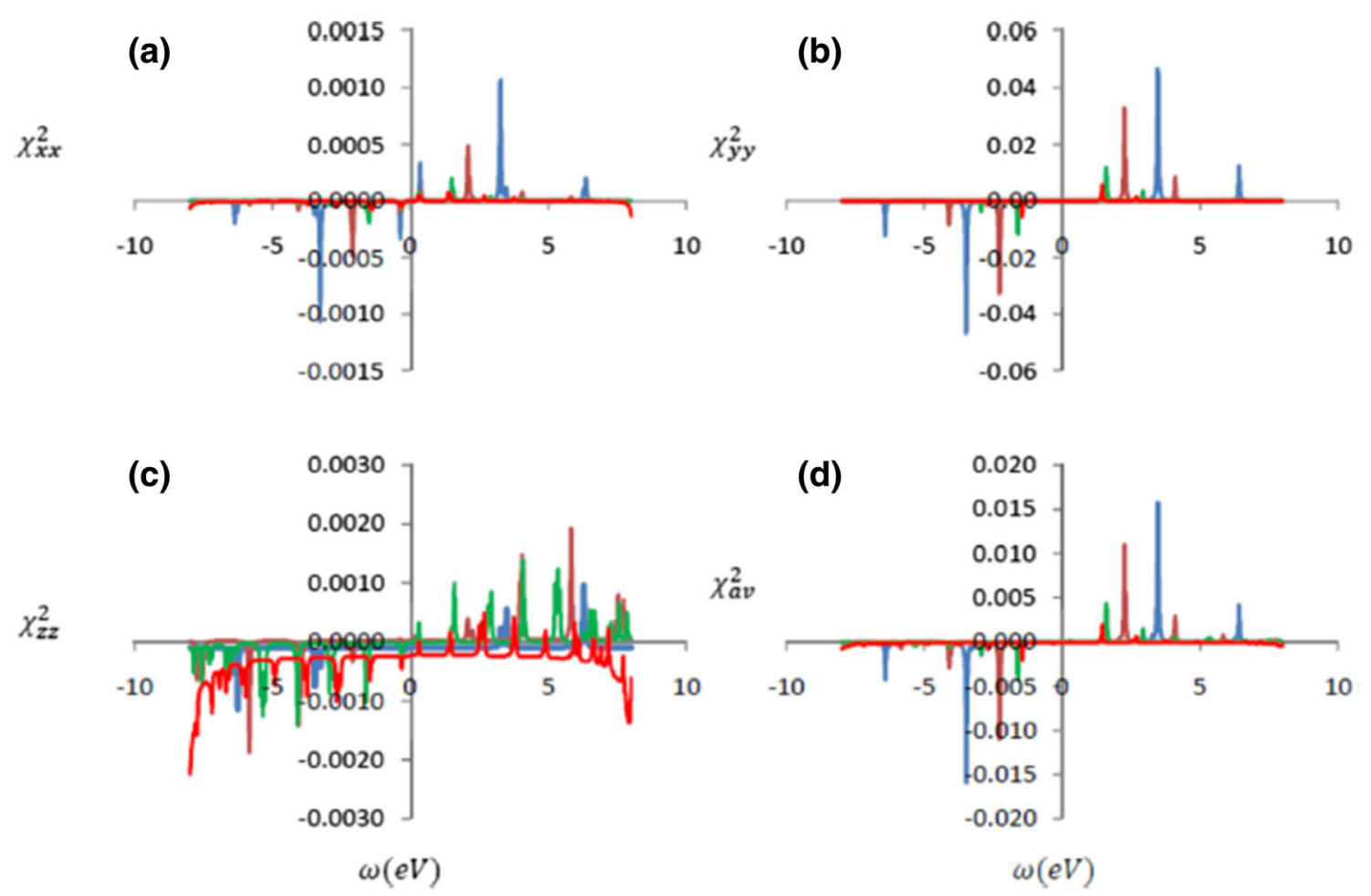

Fig. 4 The imaginary part of the a $x x$, b $y y, \mathbf{c} z z$ elements of the susceptibility tensor, and $\mathbf{d}$ average of the susceptibility tensor for the (5, 5)@(10,10) double-walled carbon nanotubes with $\mathrm{t}-\mathrm{J}$ model in the strong correlation (Color figure online)

pancy. In the strong correlation $\left(\frac{U}{t}>1\right)$, the calculated real and imaginary parts of the $x x, y y$ and $z z$ elements of the susceptibility tensor that are denoted by $\chi_{i j}^{1}$ and $\chi_{i j}^{2}$, respectively, are shown in Figs. 3a-c and 4a-c for the $(5,5) @(10,10)$ double-walled carbon nanotubes. Our calculations indicate that the $\chi_{x x}, \chi_{y y}$ and $\chi_{z z}$ spectra are different. This is due to a strong anisotropy of DWCNT. Then, the susceptibility tensor elements vary depending on the DWCNT orientation relative to the electric field of light. The imaginary part of the susceptibility tensor could exhibit the pronounced peaks at the specific frequencies, as shown in Fig. 4a-d. Such peaks are due to the single-particle excitations of the band-edge states. On the other hand, the real part of the susceptibility tensor exhibits drastic changes there. The intertube atomic overlaps, which lead to the overlaps of valence and conduction bands, account for the complicated excitation spectrum. The first peak of the susceptibility spectrum of the $(5,5) @(10,10)$ double-walled carbon nanotubes with $\mathrm{t}-\mathrm{J}$ model centered at $\omega=0.26$ (in $\left.\frac{U}{t}=1\right), 0.35$ (in $\frac{U}{t}=2$ ), 0.3 (in $\frac{U}{t}=4$ ) and $0.29 \mathrm{eV}$ (in $\frac{U}{t}=5$ ) when the electric field is in the direction $x, \omega=3.48$ (in $\left.\frac{U}{t}=1\right), 2.26$ (in $\frac{U}{t}=2$ ), 1.6 (in $\frac{U}{t}=4$ ) and $1.44 \mathrm{eV}\left(\right.$ in $\left.\frac{U}{t}=5\right)$ when the electric field is in the direction $y, \omega=3.26$ (in $\frac{U}{t}=1$ ), 2.08 (in $\frac{U}{t}=2$ ), 0.3 (in $\left.\frac{U}{t}=4\right)$ and $0.3 \mathrm{eV}\left(\right.$ in $\frac{U}{t}=5$ ) when the electric field is in the direction $z$ in Figs. 3 and 4. We calculated the average real $\chi_{a v}^{1}$ and imaginary $\chi_{a v}^{2}$ parts of the elements of the sus-

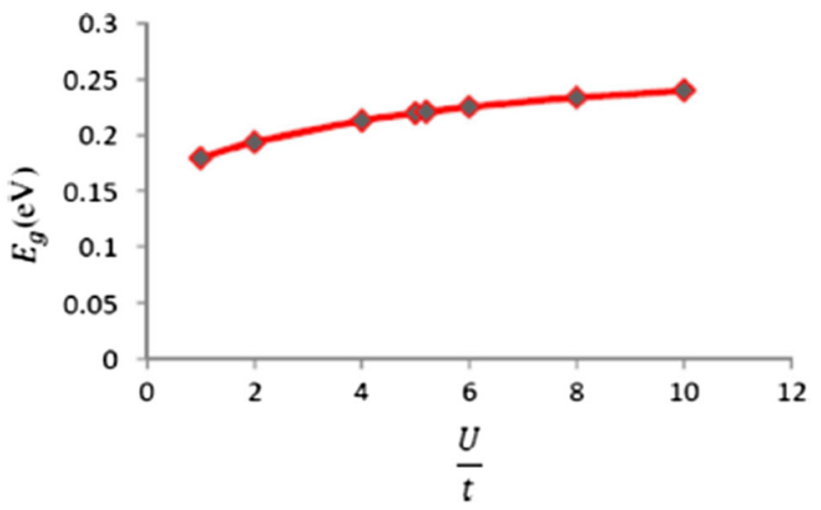

Fig. 5 Optical gap of the $(5,5) @(10,10)$ double-walled carbon nanotubes as a function of the Hubbard parameter with $\mathrm{t}-\mathrm{J}$ model in the strong correlation $\left(\frac{U}{t} \geq 1\right)$

ceptibility tensor and our results are shown in Figs. 3d and $4 \mathrm{~d}$ that the first peak centered at $\omega=3.47$ (in $\frac{U}{t}=1$ ), 2.26 (in $\frac{U}{t}=2$ ), 1.6 (in $\frac{U}{t}=4$ ) and $1.44 \mathrm{eV}$ (in $\frac{U}{t}=5$ ). Our results show that the susceptibility tensor is shifted to lower energies with increasing $\mathrm{U}$. The evolution of the optical gap of the $(5,5) @(10,10)$ double-walled carbon nanotubes as a function of the Hubbard parameter with $\mathrm{t}-\mathrm{J}$ model in the strong correlation $\left(\frac{U}{t} \geq 1\right)$ is shown in Fig. 5. The optical gap increases with increasing U. Semiempirical configuration interaction calculations reveal excitonic electronic structures 
for SWCNTs. Corresponding to each band-to-band transition within TB theory, there occurs an optical exciton in SWCNTs. The ratio problem is a simple consequence of nearly equal blueshifts of the two lowest optical absorptions from their TB frequencies. The binding energies of the lowest two excitons decrease with increasing diameter and the two binding energies are comparable for wide NTs. Assumption of similar Coulomb parameters in SWCNTs and phenyl-based $\pi$-conjugated polymers gives smaller binding energy for the former. They estimate $0.3-0.5 \mathrm{eV}$ binding energy for the wide SWCNTs. H. Zhao and S. Mazumdar ascribe the low quantum efficiency of the photoluminescence in SWCNTs to the occurrence of optically forbidden excitons below the optical exciton, which in turn is a consequence of the splitting of the degeneracy that exists in the one-electron limit by $e-e$ interactions. A similar degeneracy splitting should also occur between the states to which optical excitations transverse to the NT axis occurs [33]. Optical absorption spectra are an efficient monitor of the DWCNT sample quality and of the diameter distributions of the inner and outer DWCNT shells. Besides, they provide a valuable guide for choosing an appropriate excitation in Raman measurements [34]. For the $(5,0)$ nanotube, Alfonsi et al. found the dark states at 119, 290 and $308 \mathrm{meV}$ [35]. These are values of the order of those found in experiments by Kiowski et al. (40 and $110 \mathrm{meV}$ ) [36] and Harutyunyan et al. (30-60 and 110-190 meV) [37]. Optical absorption measurements of sorted DWCNTs reveal outer-wall purities of 96 and $98 \%$ for sorted semiconducting and metallic samples, respectively. Electrical characterization of semiconducting and metallic outer-wall DWCNTs in thin film transistors directly confirms the efficacy of these separations, with semiconducting DWCNT devices yielding on/off ratios 2 orders of magnitude higher than comparable metallic DWCNT devices [38].

\section{Conclusions}

These results clearly show that electronic correlations effects are important, and should be taken into account in theoretical studies of DWCNTs. In the weak correlation, the spin susceptibility is larger than the charge susceptibility for the $(5,5) @(10,10)$ double-walled carbon nanotubes. Also, in the strong correlation, the susceptibility tensor is shifted to lower energies with increasing U. The intertube atomic overlaps of the armchair DWCNTs significantly affects the low-energy electronic properties, such as the shift of the Fermi level, the destruction of the band symmetry, the hybridization of the wave functions, and the overlaps of the valence and conduction bands. Such drastic changes would be reflected directly in the low-frequency electronic excitations. The intertube atomic overlaps would largely reduce the plasmon strength, produce more plasmon modes, and make acoustic plasmons change into optical plasmons. The optical plasmons are directly related to free holes in valence bands and free electrons in conduction bands.

Open Access This article is distributed under the terms of the Creative Commons Attribution 4.0 International License (http://creativecomm ons.org/licenses/by/4.0/), which permits unrestricted use, distribution, and reproduction in any medium, provided you give appropriate credit to the original author(s) and the source, provide a link to the Creative Commons license, and indicate if changes were made.

\section{References}

1. Shimada, T., Sugai, T., Ohno, Y., Kishimoto, S., Mizutani, T., Yoshida, H., Okazaki, T., Shinohara, H.: Double-wall carbon nanotube field-effect transistors: ambipolar transport characteristics. Appl. Phys. Lett. 84, 2412-2414 (2004)

2. Li, Y.F., Hatakeyama, R., Kaneko, T., Izumida, T., Okada, T., Kato, T.: Electronic transport properties of Cs-encapsulated doublewalled carbon nanotubes. Appl. Phys. Lett. 89, 093110-093112 (2006)

3. Liu, K., Wang, W., Xu, Z., Bai, X., Wang, E., Yao, Y., Zhang, J., Liu, Z.: Properties and applications of single-, double- and multi-walled carbon nanotubes. J. Am. Chem. Soc. 131, 62-63 (2008)

4. Flahaut, E., Bacsa, R., Peigney, A., Laurent, C.: Gram-scale CCVD synthesis of double walled carbon nanotubes. Chem. Commun. 12, 1442-1444 (2003)

5. Endo, M., Muramatsu, H., Hayashi, T., Kim, Y.A., Terrones, M., Dresselhaus, M.S.: Nanotechnology: 'Buckypaper'from coaxial nanotubes. Nature 433, 476-476 (2005)

6. Green, A.A., Hersam, M.C.: Processing and properties of highly enriched double-wall carbon nanotubes. Nature Nanotechnol. 4, 64-70 (2009)

7. Saito, R., Matsuo, R., Kimura, T., Dresselhaus, G., Dresselhaus, M.S.: Anomalous potential barrier of double-wall carbon nanotube. Chem. Phys. Lett. 348, 187-193 (2001)

8. Chen, J., Yang, L., Yang, H., Dong, J.: Electron transport properties of incommensurate double-walled carbon nanotubes. Chem. Phys. Lett. 400, 384-388 (2004)

9. Liang, S.-D.: Intrinsic properties of electronic structure in commensurate double-wall carbon nanotubes. Phys. B. 352, 305-311 (2004)

10. Song, W., Ni, M., Lu, J., Gao, Z., Nagase, S., Yu, D., Ye, H., Zhang, $\mathrm{X}$.: Electronic structures of semiconducting double-walled carbon nanotubes: important effect of interlayer interaction. fluorescence spectroscopy of single-walled carbon nanotubes synthesized from alcohol. Chem. Phys. Lett. 414, 429-433 (2005)

11. Hertel, T., Hagen, A., Talaaev, V., Arnold, K., Hennrich, F., Kappes, M., Rosenthal, S., McBride, J., Ulbricht, H., Flahaut, E.: Spectroscopy of single- and double-wall carbon nanotubes in different environments. Nano Lett. 5, 511-514 (2005)

12. Kishi, N., Kikuchi, S., Ramesh, P., Sugai, T., Watanabe, Y., Shinohara, H.: Enhanced photoluminescence from very thin double-wall carbon nanotubes synthesized by the zeolite-CCVD method. J. Phys. Chem. B. 110, 24816-24821 (2006)

13. Mintmire, J.W., Dunlap, B.I., White, C.T.: Are fullerene tubules metallic? Phys. Rev. Lett 68, 631-634 (1992)

14. Hamada, N., Sawada, S., Oshiyama, A.: New one-dimensional conductors: graphitic microtubules. Phys. Rev. Lett. 68, 1579-1581 (1992)

15. Saito, R., Fujita, M., Dresselhaus, G., Dresselhaus, M.S.: Electronic structure of chiral graphene tubules. Phys. Rev. B 45, 6234-6242 (1992) 
16. Kwon, Y.-K., Saito, S., Toma'nek, D.: Effect of interube coupling on the electronic structure of carbon nanotube ropes. Phys. Rev. B 58, R13314-R13317 (1998)

17. Delaney, P., Choi, H.J., Ihm, J., Louie, S.G., Cohen, M.L.: Broken symmetry and pseudogaps in ropes of carbon nanotubes. Phys. Rev. B 60, 7899-7904 (1999)

18. Heeger, A.J., Kivelson, B., Schrieffer, J.R., Su, S.P.: Solitons in conducting polymers. Rev. Mod. Phys. 60, 781-850 (1988)

19. Lin, F., Sørensen, E.S., Kallin, C., Berlinsky, A.J.: Strong correlation effects in the fullerene $\mathrm{C} 20$ studied using a one-band Hubbard model. Phys. Rev. B 76, 033414-4 (2007)

20. Dagotto, E.: The t-J and frustrated Heisenberg model: a status report on numerical studies. Int. J. Mod. Phys B5, 907-935 (1991)

21. Scalettar, R.T., Dagotto, E., Bergomi, L., Jolicoeur, T., Monien, H.: Ground state properties of the hubbard model on a C60 cluster. Phys. Rev. B 47, 12316-12319 (1993)

22. Coffey, D., Trugman, S.A.: Magnetic properties of undoped C60. Phys. Rev. Lett. 69, 176-179 (1992)

23. Lovey, D.A., Romero, R.H.: Quantum transport through single and multilayer icosahedral fullerenes. J. Appl. Phys. 114, 144305144307 (2013)

24. Liu, F., Liu, C., Wu, K., Yang, F., Yao, Y.: d+id' chiral superconductivity in bilayer silicene. Phys. Rev. Lett. 111, 066804-066814 (2013)

25. Ma, J., Yuan, R.: Electronic and optical properties of finite zigzag carbon nanotubes with and without Coulomb interaction. Phys. Rev. B 57, 9343-9348 (1998)

26. Nemanich, R.J., Solin, S.A.: First- and second-order Raman scattering from finite-size crystals of graphite. Phys. Rev. B. 20, 392-401 (1979)

27. Benoit, J.M., Buisson, J.P., Chauvet, O., Godon, C., Lefrant, S.: Experiments and theory low-frequency Raman studies of multiwalled carbon nanotubes. Phys. Rev. B. 66, 073417-4 (2002)

28. Bacsa, R.R., Peigney, A., Laurent, Ch., Puech, P., Bacsa, W.S.: Chirality of internal metallic and semiconducting carbon nanotubes. Phys. Rev. B. 65, 161404-4(R) (2002)

29. Bandow, S., Chen, G., Sumanasekera, G.U., Gupta, R., Yudasaka, M., Lijima, S., Eklund, P.C.: Diameter-selective resonant Raman scattering in double-wall carbon nanotubes. Phys. Rev. B 66, 075416-075418 (2002)
30. Simon, F., Kramberger, Ch., Pfeiffer, R., Kuzmany, H., Zolyomi, V., Kurti, J., Singer, P.M., Alloul, H.: Isotope engineering of carbon nanotube systems. Phys. Rev. Lett. 95, 017401-017404 (2005)

31. Lin, F., Sorensen, E.S.: Estimates of effective Hubbard model parameters for $\mathrm{C}_{20}$ isomers. Phys. Rev. B 78, 085435-085436 (2008)

32. Konstantinidis, N.P.: Ground state magnetic response of two coupled dodecahedra. Phys. Rev. B 72, 064453-11 (2005)

33. Zhao, H., Mazumdar, S.: Electron-electron interaction effects on the optical excitations of semiconducting single-walled carbon nanotubes. Phys. Rev. Lett. 93, 157402-157404 (2004)

34. Iakoubovskii, K., Minami, N., Ueno, T., Kazaoui, S., Kataura, H.: Optical characterization of double-wall carbon nanotubes: evidence for inner tube shielding. J. Phys. Chem. C 112(30), 11194-11198 (2008)

35. Alfonsi, J., Lanzani, G., Meneghetti, M.: Exact diagonalization of Hubbard models for the optical properties of single-wall carbon nanotubes. N. J. Phys. 12, 083009-9 (2010)

36. Kiowski, O., Arnold, K., Lebedkin, S., Hennrich, F., Kappes, M.: Direct observation of deep excitonic states in the photoluminescence spectra of single-walled carbon nanotubes. Phys. Rev. Lett. 99, 237402-237404 (2007)

37. Harutyunyan, H., Gorkus, T., Green, A.A., Hersam, M.C., Allegrini, M., Hartschuh, A.: Defect induced photoluminescence from dark excitonic states in individual single-walled carbon nanotubes. Nano. Lett. 9(5), 2010-2014 (2009)

38. Green, Alexander A., Hersam, Mark C.: Properties and application of double-walled carbon nanotubes sorted by outer-wall electronic type. Acs Nano 5(2), 1459-1467 (2011) 